\title{
Development and Validation of a MicroRNA Panel to Differentiate Between Patients with Rheumatoid Arthritis or Systemic Lupus Erythematosus and Controls
}

\author{
Michelle J. Ormseth (D), Joseph F. Solus, Quanhu Sheng (1), Fei Ye (i), Qiong Wu, Yan Guo (D), \\ Annette M. Oeser, Ryan M. Allen ${ }^{\mathbb{D}}$, Kasey C. Vickers, and C. Michael Stein
}

\begin{abstract}
Objective. MicroRNA (miRNA) are short noncoding RNA that regulate genes and are both biomarkers and mediators of disease. We used small RNA (sRNA) sequencing and machine learning methodology to develop an miRNA panel to reliably differentiate between rheumatoid arthritis (RA) or systemic lupus erythematosus (SLE) and control subjects.

Methods. Plasma samples from 167 RA and 91 control subjects who frequency-matched for age, race, and sex were used for sRNA sequencing. TIGER was used to analyze miRNA. DESeq2 and random forest analyses were used to identify a prioritized list of miRNA differentially expressed in patients with RA. Prioritized miRNA were validated by quantitative PCR, and lasso and logistic regression were used to select the final panel of 6 miRNA that best differentiated RA from controls. The panel was validated in a separate cohort of 12 SLE, 32 RA, and 32 control subjects. Panel efficacy was assessed by area under the receiver operative characteristic curve (AUC) analyses.

Results. The final panel included miR-22-3p, miR-24-3p, miR-96-5p, miR-134-5p, miR-140-3p, and miR-627-5p. The panel differentiated RA from control subjects in discovery (AUC $=0.81$ ) and validation cohorts $(A U C=0.71)$, seronegative RA $(A U C=0.84)$, RA remission $(A U C=0.85)$, and patients with SLE (AUC $=0.80$ ) versus controls. Pathway analysis showed upstream regulators and targets of panel miRNA are associated with pathways implicated in RA pathogenesis.

Conclusion. An miRNA panel identified by a bioinformatic approach differentiated between RA or SLE patients and control subjects. The panel may represent an autoimmunity signature, perhaps related to inflammatory arthritis, which is not dependent on active disease or seropositivity. (First Release September 15 2019; J Rheumatol 2020;47:188-96; doi:10.3899/jrheum.181029)
\end{abstract}

Key Indexing Terms:

RHEUMATOID ARTHRITIS

BIOLOGICAL MARKERS

AUTOIMMUNITY
DISEASE ACTIVITY

From the Tennessee Valley Healthcare System, US Department of Veterans Affairs; Department of Medicine, Vanderbilt University Medical Center; Department of Biostatistics, Vanderbilt University Medical Center, Nashville, Tennessee; Department of Bioinformatics, University of New Mexico, Albuquerque, New Mexico, USA.

Funded by the Veterans Health Administration CDA IK2CX001269, Arthritis Foundation Delivering on Discovery grant, Alpha Omicron Pi, US National Institutes of Health grants P60 AR056116, P01 HL116263, and CTSA award UL1TR000445 from the National Center for Advancing Translational Sciences.

M.J. Ormseth, MD, Tennessee Valley Healthcare System, US Department of Veterans Affairs, and Department of Medicine, Vanderbilt University Medical Center; J.F. Solus, PhD, Department of Medicine, Vanderbilt University Medical Center; Q. Sheng, PhD, Department of Biostatistics, Vanderbilt University Medical Center; F. Ye, PhD, Department of Biostatistics, Vanderbilt University Medical Center; $Q$. Wu, PhD, Department of Medicine, Vanderbilt University Medical Center; Y. Guo, PhD, Department of Bioinformatics, University of New Mexico; A.M. Oeser, BA, MLAS, CCRP, Department of Medicine, Vanderbilt University Medical Center; R.M. Allen, PhD, Department of Medicine, Vanderbilt University Medical Center;

K.C. Vickers, PhD, Department of Medicine, Vanderbilt University Medical Center; C.M. Stein, MBChB, Department of Medicine, Vanderbilt University Medical Center.

Address correspondence to Dr. M.J. Ormseth, 116121 st Ave. South, T-3113 MCN, Nashville, Tennessee 37232-2681, USA.

E-mail:michelle.ormseth@vumc.org

Accepted for publication May 1, 2019.
MicroRNA (miRNA) are small noncoding RNA that are important gene regulators and serve as biomarkers of disease, because gene regulators miRNA can destabilize messenger RNA (mRNA) and block translation typically by binding to the $3^{\prime}$-untranslated region of the mRNA with a complementary seed region near the miRNA $5^{\prime}$ end ${ }^{1,2}$. MiRNA are found within cells, but also circulate in plasma protected from degradation by exosomes ${ }^{3}$, microvesicles ${ }^{4}$, lipoproproteins ${ }^{5}$, and RNA-binding proteins ${ }^{6}$. Moreover, miRNA within these bodies can be transported to recipient cells ${ }^{5}$ to regulate genes. Plasma miRNA are stable in stored samples ${ }^{7}$ and are more practical for use as biomarkers than miRNA in specific cell types owing to ease of isolation. Many studies, predominantly in cancer, show that miRNA can be helpful diagnostic and prognostic biomarkers, particularly when used in a panel composed of multiple miRNA ${ }^{8,9,10,11}$.

We and others have found several plasma miRNA that are differentially altered among patients with rheumatoid arthritis $(\mathrm{RA})^{12,13,14}$. However, most prior studies examined a few miRNA with known relevant function using PCR or small arrays. Small RNA sequencing makes it possible to evaluate

Personal non-commercial use only. The Journal of Rheumatology Copyright $($ C 2020. All rights reserved. 
many more miRNA in an unbiased fashion. Thus, small RNA sequencing could reveal novel miRNA signatures of RA and potentially provide mechanistic insights into disease pathogenesis. Our objective was to determine whether a panel of miRNA derived from small RNA sequencing could differentiate between patients with RA and control subjects, and if that panel validated in a separate RA cohort and was unique to RA or shared with another autoimmune disease: systemic lupus erythematosus (SLE). Additionally, we used pathway analysis to evaluate whether these miRNA have common disease-related upstream regulators that could affect their expression and whether the miRNA could affect RA-related pathways.

\section{MATERIALS AND METHODS}

Overview. We used a discovery cohort of RA and control subjects to perform small RNA sequencing for identification of candidate panel miRNA. Potential candidate miRNA differentiating RA from controls were prioritized using univariable differential expression analysis (R package DESeq2) and a multivariable random forest analysis. These prioritized candidates were validated by quantitative PCR (qPCR). Then, cross-validation lasso with logistic regression was used to further reduce validated miRNA candidates to a small panel of miRNA that provided best discrimination between RA and control subjects based on area under the receiver operating characteristic curves (AUC). This reduced panel of miRNA was then externally validated in an independent cohort of patients with RA and control subjects by qPCR. The panel was also tested in a small number of patients with SLE to see whether it similarly differentiated between SLE and control subjects.

Study population. The discovery cohort included 167 patients with RA and 91 control subjects who were frequency-matched for age, race, and sex from a prior cross-sectional study ${ }^{15}$. The validation cohort included 32 patients with RA and 32 control subjects who were frequency-matched for age, race, and sex from another prior cross-sectional study ${ }^{16}$, and 12 patients with SLE from another prior study ${ }^{17}$.

Recruitment and study procedures were described previously ${ }^{15,16,17}$ and will be detailed briefly. For the discovery and validation cohorts, subjects were 18 years of age or older. Patients with RA met classification criteria for RA ${ }^{18}$, patients with SLE met classification criteria for $\mathrm{SLE}^{19}$, and control subjects did not have a diagnosis of inflammatory autoimmune disease. Additionally, in the RA validation cohort, which was originally studied to examine the relationship between RA and structural and functional cardiac abnormalities, these participants were excluded from the study: those with current or prior heart failure, ischemic cardiovascular disease, structural cardiac disease, atrial fibrillation, estimated glomerular filtration rate $<60$ $\mathrm{ml} / \mathrm{min}$, and gadolinium hypersensitivity, and those who were pregnant or breastfeeding, or unable to have magnetic resonance imaging ${ }^{16}$. Studies were approved by the Vanderbilt Institutional Review Board (IRB\# 000567 , 120314, and 990111) and all subjects gave written informed consent.

Clinical and laboratory information. We collected clinical information and laboratory measurements as previously described ${ }^{15,16}$. RA disease activity was determined by the 28-joint Disease Activity Score (DAS28) using the erythrocyte sedimentation rate $(\mathrm{ESR})^{20}$. High-sensitivity C-reactive protein (CRP) concentrations and ESR were measured by the Vanderbilt University Medical Center Clinical Laboratory.

Details of sRNA sequencing and microRNA alignments. Total RNA was extracted from stored plasma using Total RNA Purification Kits (Norgen). Libraries were prepared using TruSeq Small RNA Library Preparation Kits (Illumina). RNA extractions and library preparations were performed with both RA and control subject samples in each batch. Libraries were assessed for quality and size-selected for about 128 to 157 nucleotides in length including adaptors by Pippin Prep (Sage Science) in the Vanderbilt Technologies for Advanced Genomics (VANTAGE) core facility.
The cDNA libraries were sequenced using an Illumina NextSeq500 instrument by the VANTAGE core facility. TIGER (Tools for Integrative Genome analysis of Extracellular sRNA), an in-house small RNA sequencing analysis pipeline ${ }^{21}$, was used to analyze sequence data. In brief, high-quality reads were demultiplexed using Illumina's CASAVA 1.8 pipeline and 3' adapters were trimmed using Cutadapt ${ }^{22}$. Reads shorter than 16 nucleotides after adapter trimming were discarded. Three non-templated nucleotide addition (NTA) isoforms of each sRNA read were generated by removing 1, 2, or 3 bases from the 3' terminal. All 4 isoforms, including 3 NTA isoforms and original read, were aligned to the human genome (hg19) using Bowtie ${ }^{23}$, allowing 1 mismatch. An sRNA read was identified as an miRNA if its mapped starting position matched any of the first 3 positions from the 5' end of an miRNA based on the miRNA genome coordinates from miRBase (version 21, http://www.mirbase.org).

MicroRNA analyses. To obtain a prioritized group of miRNA that were potential candidates for the panel, we used both DESeq2 ${ }^{24}$, which is Wald test-based but can adjust for batch effect and other covariates, and random forest analysis, which is a machine learning method, to identify a prioritized list of candidate miRNA for the panel. We assumed that many of the miRNA would overlap, but that using both would help broaden potential candidates.

Sequencing read counts were normalized to total sRNA reads sequenced, which passed quality control (reads per total read). Differentially expressed miRNA were compared by DESeq $2^{24}$, adjusting for age, race, sex, and batch effect. Benjamini-Hochberg-adjusted $\mathrm{p}$ values were used to select the 15 most significantly differentially expressed miRNA (based on $p$ value) for qPCR validation and further model development.

Random forest analyses, which allows for nonlinear relationships between disease status and miRNA, were conducted to select miRNA that are most important in separating RA and control subjects. The cross-validated prediction performance of models was evaluated with sequentially reduced number of predictors (ranked by variable importance) by a nested cross-validation procedure. Based on importance score, the top 15 miRNA were selected for $\mathrm{qPCR}$ validation and further model development.

Using qPCR-based plasma concentrations of the miRNA, we used lasso regularization with logistic regression to select a parsimonious final miRNA panel that maximized discrimination between RA and controls. This panel of miRNA was validated using $\mathrm{qPCR}$ in a separate cohort of 12 patients with SLE, 32 patients with RA, and 32 control subjects. Panel efficacy was assessed by AUC analyses.

$q P C R$ validation. The same plasma samples used for small RNA sequencing were also used for qPCR validation. A cocktail of 3 Caenorhabditis elegans miRNA mimics (cel-miR-39, cel-miR-54, and cel-miR238; Qiagen) was added after the initial lysis step as a spike-in standard for normalizing RNA extraction efficiency. cDNA was prepared using qScript microRNA cDNA synthesis kits (Quantabio). Individual PCR assays (Quanta) and PerfeCTa SYBR green supermix for iQ (Quantabio) were used for qPCR. Plasma miRNA concentrations were determined from standard dilution curves of a DNA mimic and normalized to the spike-in standard. Samples were excluded from analysis if the $\mathrm{Ct}$ values of the spike-in standard exceeded $1 \mathrm{Ct}$ from the median.

General statistical methods. Descriptive statistics were calculated as median [interquartile range] for continuous variables, and frequency and proportions for categorical variables. Wilcoxon rank-sum tests were used to compare continuous variables and Pearson chi-square test to compare categorical variables. PCR-based miRNA concentrations were log-transformed in models because of skewness and are presented as geometric mean (95\% CI). Fold difference of the PCR-based miRNA concentrations was the fold difference of the geometric mean. Spearman correlation was used to assess the correlation of continuous variables.

Sample size determination. For sRNA sequencing, the discovery cohort sample size of 167 RA and 91 control subjects offered about $99 \%$ power to detect miRNA that were $>1.5$-fold altered in RA versus control subjects, assuming detection across all samples of about 500 miRNA with a 5\% false discovery rate (FDR; https://cqs.mc.vanderbilt.edu/shiny/RnaSeqSampleSize/).

Personal non-commercial use only. The Journal of Rheumatology Copyright (C) 2020. All rights reserved. 
This sample size also gave about $99 \%$ power to detect an AUC $\geq 0.65$ based on PCR-based plasma miRNA concentrations.

For the validation cohort, a sample size of 32 patients with RA and 32 controls provided about $90 \%$ power to detect and $\mathrm{AUC} \geq 0.7$ based on PCR-based plasma miRNA concentrations.

Pathway analysis. We separately evaluated upstream regulators of the panel miRNA and downstream targets regulated by the panel miRNA using Ingenuity Pathway Analysis (Qiagen, Version 01-07). For evaluation as upstream regulators, we selected direct and indirect regulators of each mature miRNA and its precursor using all available data. For evaluation of downstream targets of the panel miRNA, a target was included in analysis if it was previously experimentally validated or is a highly predicted target of the miRNA. We assessed canonical pathways and functional analyses.

\section{RESULTS}

Clinical characteristics. The discovery cohort included 167 patients with RA and 91 control subjects. The groups were of similar age, race, and sex (Table 1). The RA validation cohort included 32 patients with RA and 32 control subjects of similar age, race, and sex (Supplementary Table 1, available with the online version of this article). Compared to the discovery cohort, disease activity was lower (DAS28 score 3.89 vs 2.80 units), and there were fewer seropositive individuals in the validation cohort (69\% vs $54 \%$ positive for rheumatoid factor). The SLE validation cohort included 12 patients with SLE (Supplementary Table 2).

Significantly altered miRNA based on sRNA sequencing comparing RA versus control subjects: discovery cohort. Among the 262 reliably detected plasma miRNA, 175 were significantly altered in RA compared to control subjects after adjusting for age, race, sex, and batch and FDR. Among these, 110 were $\geq 1.5$-fold altered in RA compared to control subjects (Figure 1). Most of these miRNA were increased in RA plasma, and 1 miRNA, miR-3168, was

Table 1. Subject characteristics: discovery cohort. Data are presented as median [interquartile range] or number $(\%)$.

\begin{tabular}{lccc}
\hline Characteristics & RA, $\mathrm{n}=167$ & Control, $\mathrm{n}=91$ & $\mathrm{p}$ \\
\hline Age, yrs & $54[45,63]$ & $53[44,59]$ & 0.35 \\
Race, white & $148(89)$ & $77(85)$ & 0.49 \\
Sex, female & $114(68)$ & $57(63)$ & 0.36 \\
Disease duration, yrs & $3[2,18]$ & - & - \\
DAS28 score, units & $3.89[2.63,4.9]$ & - & - \\
Tender joints & $2[0,7]$ & - & - \\
Swollen joints & $3[0,8]$ & - & - \\
ESR, mm/h & $16[7,36]$ & - & - \\
hsCRP, mg/l & $4[1.2,11.0]$ & $0.5[0.2,1.7]$ & -0.001 \\
RF-positive & $115(69)$ & - & - \\
Methotrexate & $117(71)$ & - & - \\
Leflunomide & $29(17)$ & - & - \\
Hydroxychloroquine & $42(25)$ & $2(2)$ & $<0.001$ \\
Corticosteroids & $89(53)$ & - & - \\
Anti-TNF- $\alpha$ & $33(20)$ & & - \\
\hline
\end{tabular}

DAS28: Disease Activity Score on 28 joints using ESR; ESR: erythrocyte sedimentation rate; hsCRP: high-sensitivity C-reactive protein; RF: rheumatoid factor (data available on 28 patients with RA); anti-TNF- $\alpha$ : anti-tumor necrosis factor- $\alpha$. significantly decreased $(-1.73$-fold decreased, $\mathrm{p}=5.0 \mathrm{E}-03$, Padj $=1.2 \mathrm{E}-02$ )

The top 15 differentially expressed plasma miRNA as determined each by DESeq 2 and by random forest analysis are listed in Table 2. Twelve of the 15 miRNA were common to both analytic approaches (miR-3615, miR-22-3p, miR-502-3p, miR-345-5p, miR-29c-3p, miR-221-3p, miR-140-3p, miR-30e-5p, miR-501-3p, miR-22-5p, miR-127-3p, miR-134-5p). Additionally, 3 miRNA were identified each by DESeq 2 only (miR-99b-5p, miR-130a-3p, miR-21-3p) and 3 by random forest only (miR-627-5p, miR-24-3p, miR-96-5p).

$P C R$ validation of altered miRNA. The top candidate miRNA (18 total) were measured by qPCR in the discovery cohort. Two of the miRNA (miR-502-3p and miR-501-3p) were too low in abundance to be assayed reliably by qPCR. All but 2 (miR-127-3p and miR-96-5p) of the remaining miRNA were significantly increased among patients with RA compared to control subjects using PCR-based concentrations of miRNA (Table 2).

MiRNA panel development. Using lasso variable selection with logistic regression of the qPCR-based concentrations of miRNA in the discovery cohort, the following miRNA were chosen for the panel so as to include the fewest miRNA that discriminated RA from control subjects: miR-22-3p, miR-24-3p, miR-96-5p, miR-134-5p, miR-140-3p, miR-627-5p. The panel had an AUC $=0.81$ (95\% CI 0.75-0.87, p < 0.001) for differentiating RA and control subjects. The panel was similarly robust among those with seropositive $\mathrm{RA}(\mathrm{AUC}=0.79,95 \%$ CI 0.73-0.86, $\mathrm{p}<0.001)$, seronegative RA (AUC $=0.84$, 95\% CI 0.77-0.91, p < 0.001), RA in remission (DAS28 score $<2.6^{25}, \mathrm{AUC}=0.85,95 \%$ CI $\left.0.78-0.92, \mathrm{p}<0.001\right)$, and high RA disease activity $(\mathrm{DAS} 28>5.1, \mathrm{AUC}=0.79$, 95\% CI 0.70-0.88, $\mathrm{p}<0.001)$.

The panel had similar performance across other subgroups of patients compared to control subjects. This included RA patients with disease duration $<1$ year $(n=29, \mathrm{AUC}=0.80$, 95\% CI $0.72-0.89, \mathrm{p}<0.001)$, those not taking biologic disease-modifying antirheumatic drugs (bDMARD; $\mathrm{n}=133$, $\mathrm{AUC}=0.81,95 \% \mathrm{CI} 0.75-0.87, \mathrm{p}<0.001)$, those taking bDMARD $(\mathrm{n}=33$, AUC $=0.80,95 \%$ CI $0.71-0.88$, $\mathrm{p}<0.001)$, those not taking conventional synthetic DMARD (csDMARD) or bDMARD $(\mathrm{n}=19, \mathrm{AUC}=0.90,95 \% \mathrm{CI}$ $0.83-0.96, \mathrm{p}<0.001)$, those taking any csDMARD or bDMARD $(\mathrm{n}=150$, AUC $=0.80,95 \%$ CI $0.73-0.86$, $\mathrm{p}<0.001$ ), those not receiving any csDMARD, bDMARD, or corticosteroid $(\mathrm{n}=13, \mathrm{AUC}=0.89,95 \%$ CI $0.83-0.96$, $\mathrm{p}<0.001$ ), and those taking either csDMARD or bDMARD or corticosteroid only $(n=153)$ compared to control subjects $(\mathrm{AUC}=0.80,95 \%$ CI 0.74-0.86, $\mathrm{p}<0.001)$.

Validation. The panel of 6 miRNA was robust in the separate RA validation cohort $(\mathrm{AUC}=0.71,95 \% \mathrm{CI}$ $0.58-0.84, \mathrm{p}=0.004)$. Similarly, in the validation cohort, the panel differentiated between seropositive ( $\mathrm{AUC}=0.73$, $95 \%$ CI $0.58-0.87, \mathrm{p}=0.01)$ or seronegative RA

Personal non-commercial use only. The Journal of Rheumatology Copyright (C) 2020. All rights reserved 


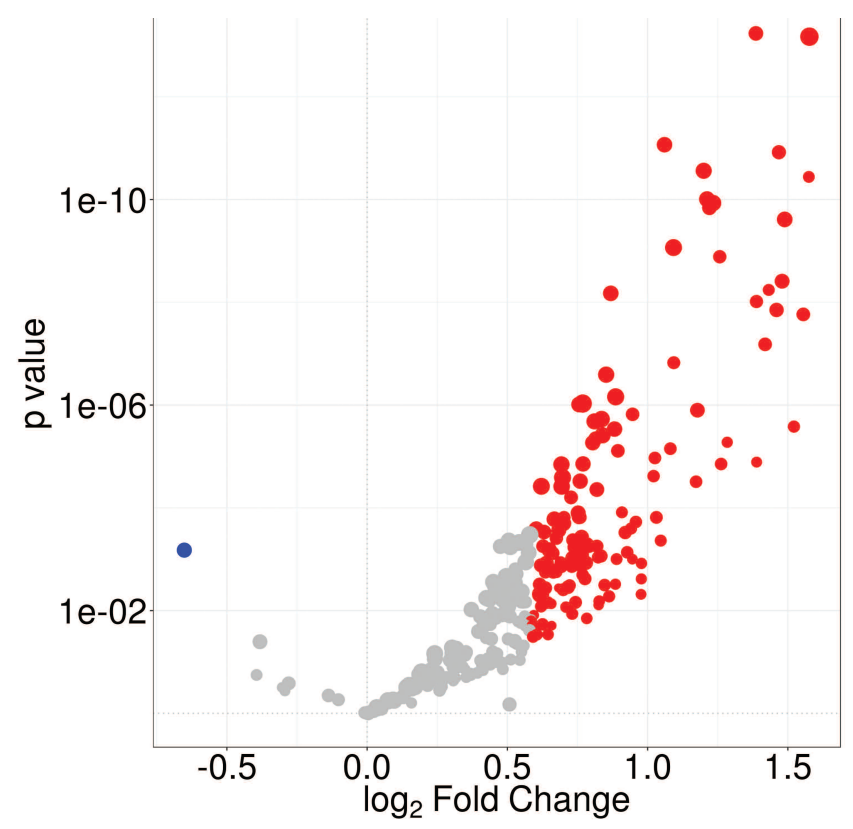

Figure 1. Volcano plot displaying differential plasma miRNA expression in the discovery cohort $(\mathrm{n}=167$ RA vs $\mathrm{n}=91$ control subjects). Small RNA sequencing was analyzed by DESeq2 and adjusted for age, race, sex, and batch and multiple comparisons. Among these, 110 were $\geq 1.5$-fold altered in RA compared to control subjects. Each dot represents an individual miRNA, and the larger the dot, the more abundant the miRNA. Red indicates increased in RA and blue indicates increased in control subjects. miRNA: microRNA; RA: rheumatoid arthritis.

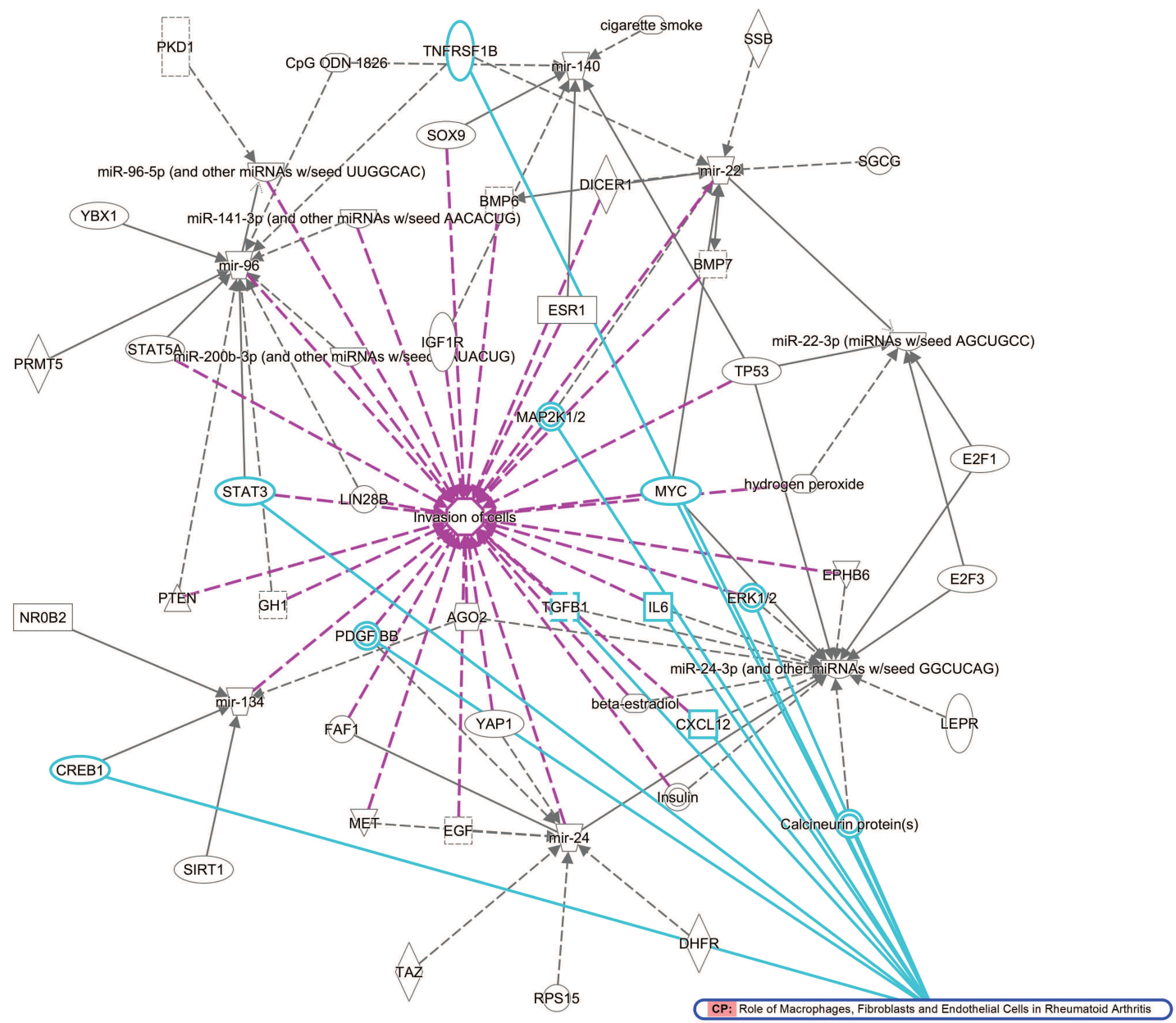

Figure 2. Upstream regulators of RA panel miRNA are involved in invasion of cells and RA-specific pathways. Using Ingenuity Pathway Analysis of upstream regulators of the mature and precursor miRNA, the top related functional pathway was related to invasion of cells and the top related canonical pathway was the role of macrophages, fibroblasts, and endothelial cells in RA MiRNA: microRNA; RA: rheumatoid arthritis. From QIAGEN Ingenuity Pathway Analysis; with permission. 
(AUC $=0.73,95 \%$ CI $0.57-0.89, \mathrm{p}=0.02)$ versus control subjects.

We additionally measured the panel in 12 patients with SLE and compared model performance to control subjects. The panel also differentiated patients with SLE from control subjects (AUC $=0.80,95 \%$ CI $0.65-0.96, p=0.001$ ), but was not significantly different comparing patients with SLE to RA (AUC $=0.63,95 \%$ CI $0.44-0.82, \mathrm{p}=0.13$ ).

Relationship between miRNA components of the panel and disease-related variables. Three of the miRNA were weakly associated with RA disease activity by DAS28 score in the discovery cohort $(\mathrm{miR}-24-3 \mathrm{p}$ : Rho $=-0.16, \mathrm{p}=0.04$; miR-96-5p: Rho $=0.16, \mathrm{p}=0.04 ; \mathrm{miR}-140-3 \mathrm{p}: \mathrm{Rho}=-0.16$, $\mathrm{p}=0.05$ ); however, these significant associations were not observed in the RA validation cohort.

Upstream regulators of these miRNA in the panel. We examined upstream regulators of the miRNA included in the panel to determine whether there are commonalities in regulation between the miRNA that would promote their ability to be used as an RA miRNA signature. There was little information regarding upstream regulators of miR-627-5p or its precursor at the time of analysis; thus this miRNA was excluded from pathway analysis. The top identified function that the upstream regulators possessed related to invasion of cells $(\mathrm{p}=7.89 \mathrm{E}-27)$, for which 35 of the upstream regulators were included (Figure 2). Additionally, these upstream regulators are involved in cell death $(\mathrm{p}=7.11 \mathrm{E}-22)$. The top overlapping canonical pathway was the involvement of macrophages, fibroblasts, and endothelial cells in RA with 11 overlapping molecules (Figure 2).

MiRNA panel pathway targets. Because circulating miRNA can be delivered to cells with functional consequences ${ }^{5}$ or could reflect cellular processes, we evaluated whether experimentally validated and highly predicted targets of panel miRNA are involved in RA pathways. There was little information regarding miR-134-5p function at the time of analysis, thus this miRNA was excluded from pathway analysis. Among the top canonical pathways, the involvement of osteoblasts, osteoclasts, and chondrocytes in RA (Supplementary Figure 1, available with the online version of this article) and the involvement of macrophages, fibroblasts, and endothelial cells in RA (Figure 3) were third (29 molecules) and sixth (28 molecules), respectively, in the number of molecules in the pathway that the miRNA panel may target. Other top canonical pathways include molecular mechanisms of cancer (52 genes), G-protein coupled receptor signaling (30 molecules), protein kinase A signaling (29 molecules), and axonal guidance signaling (28 molecules).

\section{DISCUSSION}

We used plasma sRNA sequencing and bioinformatics approaches to develop a panel of miRNA that reliably differentiates between patients with RA and control subjects. The panel included miR-22-3p, miR-24-3p, miR-96-5p, miR-134-5p, miR-140-3p, and miR-627-5p and was robust across seronegative and seropositive RA and RA of varying disease activity. The panel also differentiated between patients with SLE and control subjects but was not significantly different between patients with RA and SLE, suggesting that this panel represents an autoimmunity signature.

Strong evidence indicates that miRNA as a class of sRNA

Table 2. PCR validation of top miRNA candidates from discovery cohort sRNA sequencing.

\begin{tabular}{|c|c|c|c|c|}
\hline \multirow[t]{2}{*}{ MiRNA } & \multicolumn{2}{|c|}{ Plasma fM Concentration Presented as Geometric Mean (95\% CI) } & \multirow[t]{2}{*}{ Fold Diff } & \multirow[t]{2}{*}{$\mathrm{p}$} \\
\hline & RA & Control & & \\
\hline miR-3615 & $0.34(0.28,0.41)$ & $0.25(0.17,0.35)$ & 1.38 & 4.69E-01 \\
\hline miR-22-3p & $58.1(45.3,74.5)$ & $15.1(10.8,21)$ & 3.85 & $8.45 \mathrm{E}-12$ \\
\hline miR-502-3p & N/A & N/A & N/A & N/A \\
\hline $\mathrm{miR}-345-5 \mathrm{p}$ & $3.08 \mathrm{E}-2(1.63 \mathrm{E}-02,5.81 \mathrm{E}-02)$ & 7.75E-3 (2.95E-03, 2.04E-02) & 3.98 & $1.72 \mathrm{E}-04$ \\
\hline $\operatorname{miR}-29 c-3 p$ & $1.46(1.18,1.8)$ & $0.56(0.41,0.77)$ & 2.59 & 5.32E-09 \\
\hline miR-99b-5p & $1.50(0.86,2.61)$ & $0.99(0.48,2.05)$ & 1.51 & 7.27E-04 \\
\hline $\operatorname{miR}-221-3 p$ & $9.96(7.48,13.2)$ & $3.04(2.15,4.31)$ & 3.27 & $6.78 \mathrm{E}-09$ \\
\hline miR-140-3p & $1.22(1.0,1.49)$ & $0.25(0.17,0.37)$ & 4.93 & $2.12 \mathrm{E}-13$ \\
\hline miR-130a-3p & $5.00(3.51,7.12)$ & $1.27(0.81,1.97)$ & 3.94 & $8.56 \mathrm{E}-08$ \\
\hline miR-30e-5p & $4.23(3.51,5.11)$ & $3.24(2.49,4.21)$ & 1.31 & $2.39 \mathrm{E}-02$ \\
\hline miR-501-3p & N/A & N/A & N/A & N/A \\
\hline miR-22-5p & $1.18(0.891,1.56)$ & $0.39(0.25,0.61)$ & 2.99 & $1.25 \mathrm{E}-07$ \\
\hline miR-127-3p & $5.17(4.7,5.68)$ & $5.53(4.9,6.24)$ & -1.08 & $4.88 \mathrm{E}-01$ \\
\hline $\mathrm{miR}-21-3 \mathrm{p}$ & $1.21 \mathrm{E}-02(4.56 \mathrm{E}-03,3.23 \mathrm{E}-02)$ & $6.97 \mathrm{E}-04(1.33 \mathrm{E}-04,6.64 \mathrm{E}-03)$ & 17.4 & $4.08 \mathrm{E}-03$ \\
\hline miR-134-5p & $1.45 \mathrm{E}-01(6.38 \mathrm{E}-02,3.35 \mathrm{E}-01)$ & $6.76 \mathrm{E}-03(1.64 \mathrm{E}-03,2.80 \mathrm{E}-02)$ & 21.6 & $5.11 \mathrm{E}-07$ \\
\hline miR-627-5p & 7.18E-02 (2.99E-02, 1.72E-01) & 4.39E-03 (9.58E-04, 2.01E-02) & 16.3 & $2.73 \mathrm{E}-04$ \\
\hline $\mathrm{miR}-24-3 \mathrm{p}$ & $3.49(2.35,5.18)$ & $1.32(0.85,2.06)$ & 2.64 & $1.19 \mathrm{E}-06$ \\
\hline miR-96-5p & $13.9(12.9,14.9)$ & $15.2(13.6,17)$ & -1.10 & $1.85 \mathrm{E}-01$ \\
\hline
\end{tabular}

miRNA: microRNA; sRNA: small RNA; fM: femtomolar; fold diff: fold difference comparing the geometric mean of qPCR-based miRNA concentration in RA vs control subjects; N/A: the miRNA was too low for reliable detection by PCR; RA: rheumatoid arthritis.

Personal non-commercial use only. The Journal of Rheumatology Copyright @ 2020 . All rights reserved 


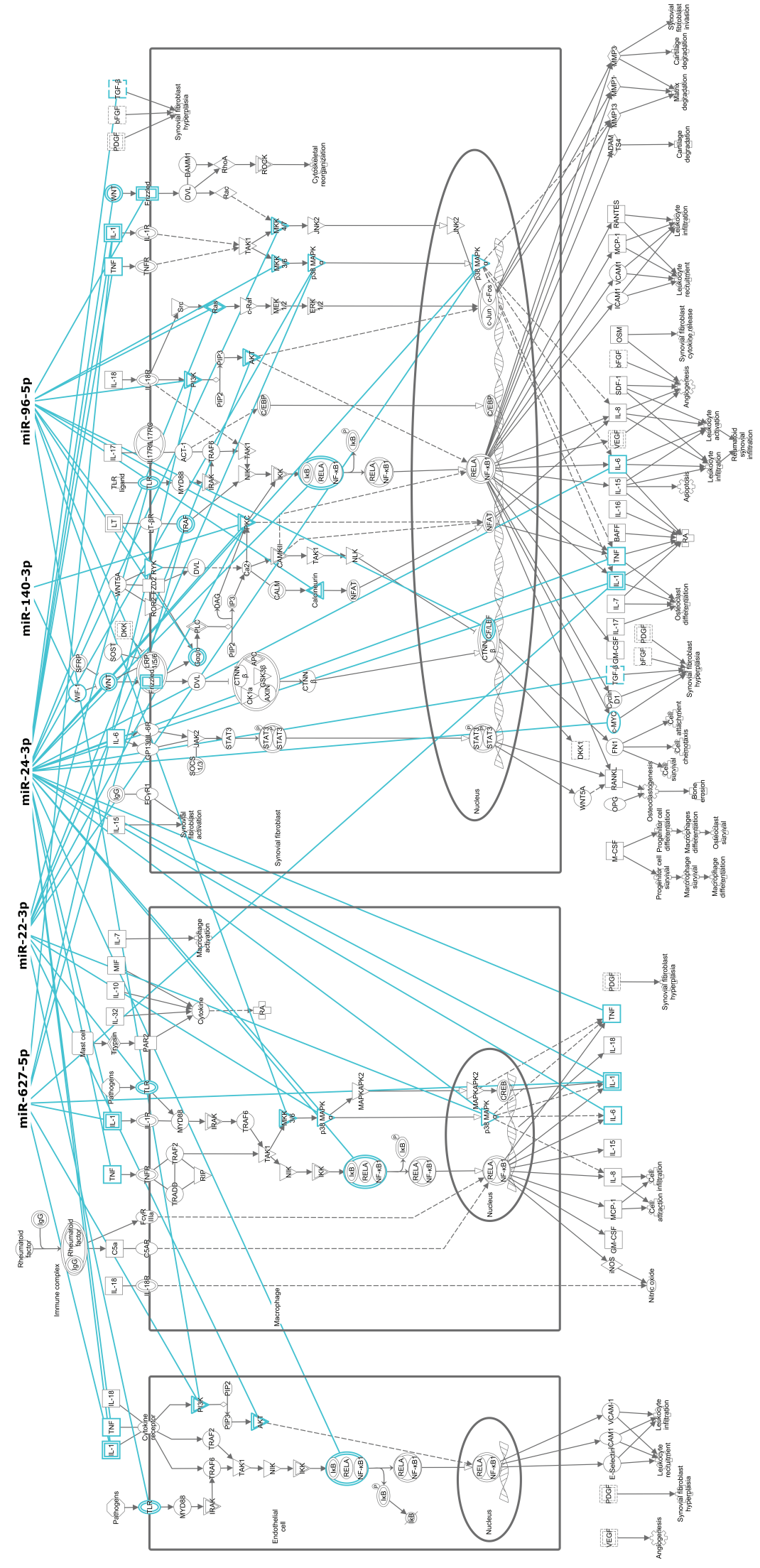

Figure 3. RA panel miRNA can target RA-specific pathways. Using Ingenuity Pathway Analysis of experimentally validated or highly predicted targets of the panel miRNA, one of the top related canonical pathways was the role of macrophages, fibroblasts, and endothelial cells in RA. miRNA: microRNA; RA: rheumatoid arthritis. 
are important in RA. Dicer and Drosha, which are endoribonucleases involved in cleaving miRNA to their mature form, are upregulated in peripheral blood mononuclear cells (PBMC) of patients with $\mathrm{RA}^{26}$. Moreover, activation of Dicer decreases tumor necrosis factor- $\alpha$ production, suggesting a homeostatic involvement of miRNA in RA. The upregulation of Dicer and Drosha in PBMC from patients with RA likely explains why we observed an overall increase in miRNA in plasma from patients with RA, because many plasma miRNA derive from PBMC and other blood cells ${ }^{27}$. However, in RA not all cell types have increased expression of Dicer and the associated increased miRNA expression. For example, Dicer and miRNA expression were lower in RA synovial fibroblasts, leading to an exaggerated response to inflammatory stimuli and resistance to apoptosis ${ }^{28}$. In addition to broad changes in miRNA production in RA, there are several individual miRNA with biologic significance implicated in RA.

Several of the panel miRNA have known associations with RA, either as biomarkers or known biology of RA. For example, miR-22-3p was increased significantly among anticitrullinated protein antibodies-positive individuals who subsequently progressed to $\mathrm{RA}^{29}$. Also, lower concentrations of miR-22-3p before treatment were associated with response to adalimumab at 12 months in a randomized double-blinded placebo-controlled trial of 180 patients with early RA ${ }^{30}$. MiR-22-3p is decreased in fibroblast-like synoviocytes (FLS) from RA compared to patients with osteoarthritis (OA); lower concentrations of miR-22-3p promoted FLS proliferation ${ }^{31}$. Thus miR-22-3p may be a driving force for the synovial hyperplasia characteristic of RA. MiR-22-3p also regulates Th17 responses in emphysema ${ }^{32}$ and drives hyperresponsive $\mathrm{B}$ cells in $\mathrm{SLE}^{33}$. Moreover, we found that inhibition of miR-22-3p improves nephritis in a mouse model of SLE (manuscript in preparation).

Plasma concentrations of miR-24-3p were elevated in patients with RA compared to controls in several prior studies, and it is a component of a prior RA plasma miRNA panel, which we and others have proposed ${ }^{12,14}$. In response to interleukin 6, miR-24-3p increases and promotes plasma cell survival, an effect that could support autoreactive plasma cells in $\mathrm{RA}^{34}$. MiR-24-3p also plays a homeostatic role by dampening inflammation through nuclear factor-KB signaling pathway ${ }^{35}$ and chitinase 3 -like- $1^{36}$ in the vasculature.

MiR-140-3p has been most widely studied in OA and participates in cartilage homeostasis ${ }^{37}$; its concentrations are also decreased in synovial tissue from patients with RA compared to $\mathrm{OA}^{38}$. Intraarticular administration of pre-miR-140 (the precursor for miR-140-3p) reduced arthritis scores in mice with collagen-induced arthritis by way of increasing synovial fibroblast apoptosis and reducing proliferation $^{38}$.

Three of the panel miRNA (miR-96-5p, miR-134-5p, and miR-627-5p) have not been studied widely in RA. Thus, if we had limited selection of miRNA for the panel to those previously studied in RA, these would have been overlooked. We identified new miRNA because of our methods that used sequencing rather than a preselected panel and random forest analysis to identify candidates, which has not been previously done in RA, to our knowledge. MiR-96-5p remained helpful to the model despite not being overall significantly altered in RA based on PCR data. Both miR-96-5p and miR-134-5p can target the KRAS signaling pathway ${ }^{39,40}$, which may affect $T$ cell activation thresholds, enabling responses to autoantigens ${ }^{41}$. Calcitriol-induced expression of miR-627 and miR-627 reduced proliferation of a colorectal cancer cell line ${ }^{42}$. Exposure of calcitrol to synoviocytes cultured from patients with RA reduced cell proliferation and cytokine production ${ }^{43}$. It is possible that miR-627 may be part of this mechanism.

Proposed pathways that promote or are altered by the miRNA in the panel. Pathway analysis indicates that upstream regulators of panel miRNA and panel miRNA targets are involved in RA-related disease processes. This observation also provides proof-of-principle for the methodology we used to develop the panel. Use of non-biased techniques, such as random forest analysis, serves to find novel biomarkers and offer some insight into mechanisms of disease.

Limitations. Both discovery and validation cohorts were predominantly patients with established disease (defined as $\geq 6$ mos of duration ${ }^{44}$ ); thus, the panel may not differentiate individuals with RA at the time of diagnosis or pre-RA states from control subjects. The panel was robust among those with disease duration $<1$ year and among those not receiving DMARD, however. We did not design the study to develop a diagnostic panel for RA when other inflammatory autoimmune diseases are under consideration, but initial testing of the panel in a small set of patients with SLE suggests that the panel may represent an autoimmunity signature. Future studies examining the panel in a variety of inflammatory autoimmune diseases at varied levels of activity would be helpful to determine whether these miRNA compose an inflammatory autoimmune disease signature rather than an RA signature. The strong relationship between the miRNA and annotated RA pathways was reassuring, but there are limitations to pathway analysis. In general, RA has more extensive annotated pathways than many other inflammatory autoimmune diseases; therefore it is possible that we do not see as strong a relationship with other inflammatory autoimmune diseases because there are insufficient annotated pathways.

An miRNA panel identified by bioinformatics approaches was able to differentiate between patients with RA and control subjects with reproducibility. Many of the upstream regulators of the miRNA and many of the miRNA targets regulate RA-related pathways. The panel may represent an autoimmunity signature that is not dependent on active disease.

Personal non-commercial use only. The Journal of Rheumatology Copyright $\odot$ 2020. All rights reserved 


\section{ONLINE SUPPLEMENT}

Supplementary material accompanies the online version of this article.

\section{REFERENCES}

1. Baek D, Villén J, Shin C, Camargo FD, Gygi SP, Bartel DP. The impact of microRNAs on protein output. Nature 2008;455:64-71.

2. Bartel DP. MicroRNAs: Target recognition and regulatory functions. Cell 2009;136:215-33.

3. Valadi H, Ekström K, Bossios A, Sjostrand M, Lee JJ, Lotvall JO. Exosome-mediated transfer of miRNAs and microRNAs is a novel mechanism of genetic exchange between cells. Nat Cell Biol 2007;9:654-9.

4. Hunter MP, Ismail N, Zhang X, Aguda BD, Lee EJ, Yu L, et al. Detection of microRNA expression in human peripheral blood microvesicles. PLoS One 2008;3:e3694.

5. Vickers KC, Palmisano BT, Shoucri BM, Shamburek RD, Remaley AT. MicroRNAs are transported in plasma and delivered to recipient cells by high-density lipoproteins. Nat Cell Biol 2011;13:423-33 .

6. Arroyo JD, Chevillet JR, Kroh EM, Ruf IK, Pritchard CC, Gibson $\mathrm{DF}$, et al. Argonaute 2 complexes carry a population of circulating microRNAs independent of vesicles in human plasma. Proc Natl Acad Sci U S A 2011;108:5003-8.

7. Mitchell PS, Parkin RK, Kroh EM, Fritz BR, Wyman SK, Pogosova-Agadjanyan EL, et al. Circulating microRNAs as stable blood-based markers for cancer detection. Proc Natl Acad Sci U S A 2008;105:10513-8.

8. Sun X, Zhou X, Zhang Y, Zhu X, Liu H. Systematic review and meta-analysis of diagnostic accuracy of miRNAs in patients with pancreatic cancer. Dis Markers 2018;2018:6292396.

9. Wang H, Peng R, Wang J, Qin Z, Xue L. Circulating microRNAs as potential cancer biomarkers: the advantage and disadvantage. Clin Epigenetics 2018;10:59.

10. Schwarzenbach H, Nishida N, Calin GA, Pantel K. Clinical relevance of circulating cell-free microRNAs in cancer. Nat Rev Clin Oncol 2014;11:145-56.

11. Alevizos I, Illei GG. MicroRNAs as biomarkers in rheumatic diseases. Nat Rev Rheumatol 2010;6:391-8.

12. Ormseth MJ, Solus JF, Vickers KC, Oeser AM, Raggi P, Stein CM. Utility of select plasma microRNA for disease and cardiovascular risk assessment in patients with rheumatoid arthritis. J Rheumatol 2015;42:1746-51.

13. Long H, Wang X, Chen Y, Wang L, Zhao M, Lu Q. Dysregulation of microRNAs in autoimmune diseases: pathogenesis, biomarkers and potential therapeutic targets. Cancer Lett 2018;428:90-103.

14. Murata K, Yoshitomi H, Tanida S, Ishikawa M, Nishitani K, Ito H, et al. Plasma and synovial fluid microRNAs as potential biomarkers of rheumatoid arthritis and osteoarthritis. Arthritis Res Ther 2010;12:R86.

15. Chung CP, Oeser A, Raggi P, Gebretsadik T, Shintani AK, Sokka T, et al. Increased coronary-artery atherosclerosis in rheumatoid arthritis: relationship to disease duration and cardiovascular risk factors. Arthritis Rheum 2005;52:3045-53.

16. Bradham W, Ormseth MJ, Elumogo C, Palanisamy S, Liu CY, Lawson MA, et al. Absence of fibrosis and inflammation by cardiac magnetic resonance imaging in rheumatoid arthritis patients with low to moderate disease activity. J Rheumatol 2018;45:1078-84.

17. Asanuma Y, Oeser A, Shintani AK, Turner E, Olsen N, Fazio S, et al. Premature coronary-artery atherosclerosis in systemic lupus erythematosus. N Engl J Med 2003;349:2407-15.

18. Arnett FC, Edworthy SM, Bloch DA, McShane DJ, Fries JF, Cooper NS, et al. The American Rheumatism Association 1987 revised criteria for the classification of rheumatoid arthritis. Arthritis Rheum 1988;31:315-24.

19. Tan EM, Cohen AS, Fries JF, Masi AT, McShane DJ, Rothfield NF, et al. The 1982 revised criteria for the classification of systemic lupus erythematosus. Arthritis Rheum 1982;25:1271-7.

20. Prevoo ML, van't Hof MA, Kuper HH, van Leeuwen MA, van de Putte LB, van Riel PL. Modified disease activity scores that include twenty-eight-joint counts. Development and validation in a prospective longitudinal study of patients with rheumatoid arthritis. Arthritis Rheum 1995;38:44-8.

21. Allen RM, Zhao S, Ramirez Solano MA, Zhu W, Michell DL, Wang $\mathrm{Y}$, et al. Bioinformatic analysis of endogenous and exogenous small RNAs on lipoproteins. J Extracell Vesicles 2018;7:1506198.

22. Schulte JH, Marschall T, Martin M, Rosenstiel P, Mestdagh P, Schlierf S, et al. Deep sequencing reveals differential expression of microRNAs in favorable versus unfavorable neuroblastoma. Nucleic Acids Res 2010;38:5919-28

23. Langmead B, Trapnell C, Pop M, Salzberg SL. Ultrafast and memory-efficient alignment of short DNA sequences to the human genome. Genome Biol 2009;10:R25.

24. Robinson MD, McCarthy DJ, Smyth GK. edgeR: a Bioconductor package for differential expression analysis of digital gene expression data. Bioinformatics 2010;26:139-40.

25. Fransen J, Creemers MC, van Riel PL. Remission in rheumatoid arthritis: agreement of the disease activity score (DAS28) with the ARA preliminary remission criteria. Rheumatology 2004;43:1252-5.

26. Wang S, Yuan M, Song L, Zhang X, Geng Q, Zhang H, et al. Expression of dicer in rheumatoid arthritis is associated with disease activity and balances the production of TNF- $\alpha$. Mol Med Rep 2017;16:1590-5.

27. Pritchard CC, Kroh E, Wood B, Arroyo JD, Dougherty KJ, Miyaji $\mathrm{MM}$, et al. Blood cell origin of circulating microRNAs: a cautionary note for cancer biomarker studies. Cancer Prev Res 2012;5:492-7.

28. Alsaleh G, Nehmar R, Blüml S, Schleiss C, Ostermann E, Dillenseger JP, et al. Reduced dicer1 expression bestows rheumatoid arthritis synoviocytes proinflammatory properties and resistance to apoptotic stimuli. Arthritis Rheumatol 2016;68:1839-48.

29. Ouboussad L, Hunt L, Hensor EMA, Nam JL, Barnes NA, Emery P, et al. Profiling microRNAs in individuals at risk of progression to rheumatoid arthritis. Arthritis Res Ther 2017;19:288.

30. Krintel SB, Dehlendorff C, Hetland ML, Horslev-Petersen K, Andersen KK, Junker P, et al. Prediction of treatment response to adalimumab: a double-blind placebo-controlled study of circulating microRNA in patients with early rheumatoid arthritis. Pharmacogenomics J 2016;16:141-6.

31. Lin J, Huo R, Xiao L, Zhu X, Xie J, Sun S, et al. A novel p53/microrna-22/cyr61 axis in synovial cells regulates inflammation in rheumatoid arthritis. Arthritis Rheumatol 2014;66:49-59.

32. Lu W, You R, Yuan X, Yang T, Samuel EL, Marcano DC, et al. The microRNA mir-22 inhibits the histone deacetylase hdac4 to promote th) 17 cell-dependent emphysema. Nat Immunol 2015;16:1185-94.

33. Wu XN, Ye YX, Niu JW, Li Y, Li X, You X, et al. Defective PTEN regulation contributes to $\mathrm{B}$ cell hyperresponsiveness in systemic lupus erythematosus. Sci Transl Med 2014;6:246ra99.

34. Gabler J, Wittmann J, Porstner M, Renz H, Jack HM, Abram M, et al. Contribution of microRNA 24-3p and erk $1 / 2$ to interleukin-6-mediated plasma cell survival. Eur J Immunol 2013;43:3028-37.

35. Zheng Y, Li Y, Liu G, Qi X, Cao X. MicroRNA-24 inhibits the proliferation and migration of endothelial cells in patients with atherosclerosis by targeting importin- $\alpha 3$ and regulating inflammatory responses. Exp Ther Med 2018;15:338-44.

36. Maegdefessel L, Spin JM, Raaz U, Eken SM, Toh R, Azuma J, et al. Mir-24 limits aortic vascular inflammation and murine abdominal aneurysm development. Nat Commun 2014;5:5214.

37. Zhang R, Ma J, Yao J. Molecular mechanisms of the cartilage-specific microrna-140 in osteoarthritis. Inflamm Res 2013;62:871-7.

Personal non-commercial use only. The Journal of Rheumatology Copyright $\subset$ 2020. All rights reserved. 
38. Peng JS, Chen SY, Wu CL, Chong HE, Ding YC, Shiau AL, et al. Amelioration of experimental autoimmune arthritis through targeting of synovial fibroblasts by intraarticular delivery of microRNAs 140-3p and 140-5p. Arthritis Rheumatol 2016; 68:370-81.

39. Ress AL, Stiegelbauer V, Winter E, Schwarzenbacher D, Kiesslich T, Lax S, et al. Mir-96-5p influences cellular growth and is associated with poor survival in colorectal cancer patients. Mol Carcinog 2015;54:1442-50.

40. Pan JY, Zhang F, Sun CC, Li SJ, Li G, Gong FY, et al. Mir-134: A human cancer suppressor? Mol Ther Nucleic Acids 2017;6:140-9.

41. Singh K, Deshpande P, Li G, Yu M, Pryshchep S, Cavanagh M, et al. K-RAS GTPase- and B-RAF kinase-mediated T-cell tolerance defects in rheumatoid arthritis. Proc Natl Acad Sci U S A 2012;109:E1629-37.
42. Padi SK, Zhang Q, Rustum YM, Morrison C, Guo B. MicroRNA-627 mediates the epigenetic mechanisms of vitamin D to suppress proliferation of human colorectal cancer cells and growth of xenograft tumors in mice. Gastroenterology 2013;145:437-46.

43. Huhtakangas JA, Veijola J, Turunen S, Karjalainen A, Valkealahti M, Nousiainen T, et al. 1,25(OH)2D3 and calcipotriol, its hypocalcemic analog, exert a long-lasting anti-inflammatory and anti-proliferative effect in synoviocytes cultured from patients with rheumatoid arthritis and osteoarthritis. J Steroid Biochem Mol Biol 2017;173:13-22.

44. Singh JA, Saag KG, Bridges SL Jr, Akl EA, Bannuru RR, Sullivan MC, et al; American College of Rheumatology. 2015 American College of Rheumatology guideline for the treatment of rheumatoid arthritis. Arthritis Care Res 2016;68:1-25. 